\title{
Differential Efficacy of TNF Inhibitors With or Without the Immunoglobulin Fc Portion in Rheumatoid Arthritis - The ANSWER Cohort Study
}

Yoichi Nakayama

Kyoto University Graduate School of Medicine https://orcid.org/0000-0002-8130-4350

Motomu Hashimoto ( $\square$ mohashim@kuhp.kyoto-u.ac.jp)

Kyoto University Graduate School of Medicine https://orcid.org/0000-0002-9241-060X

Kosaku Murakami

Kyoto University Graduate School of Medicine

Koichi Murata

Kyoto University Graduate School of Medicine

Masao Tanaka

Kyoto University Graduate School of Medicine

Hiromu lto

Kyoto University Graduate School of Medicine

Wataru Yamamoto

Kurashiki Sweet Hospital

Kosuke Ebina

Osaka University Graduate School of Medicine

Kenichiro Hata

Osaka Medical College

Yuri Hiramatsu

Osaka Medical College

Masaki Katayama

Osaka Red Cross Hospital

Yonsu Son

Kansai Medical University: Kansai Ika Daigaku

Hideki Amuro

Kansai Medical University: Kansai Ika Daigaku

Kengo Akashi

Kobe University Graduate School of Medicine School of Medicine: Kobe Daigaku Daigakuin Igakukei Kenkyuka Igakubu

Akira Onishi

Kobe University Graduate School of Medicine School of Medicine: Kobe Daigaku Daigakuin Igakukei Kenkyuka Igakubu

Ryota Hara

Nara Medical University

Keiichi Yamamoto

Wakayama Medical University

Koichiro Ohmura

Kyoto University Graduate School of Medicine

Shuichi Matsuda

Kyoto University Graduate School of Medicine

\section{Research article}

Keywords: Rheumatoid arthritis, rheumatoid factor, tumour necrosis factor, certolizumab-pegol, precision medicine, Fc portion

Posted Date: November 13th, 2020 
DOI: https://doi.org/10.21203/rs.3.rs-104478/v1

License: (c) (i) This work is licensed under a Creative Commons Attribution 4.0 International License. Read Full License 


\section{Abstract}

Background: Rheumatoid factor (RF) binds to the Fc portion of immunoglobulin and can bind to the Fc portion of anti-TNF monoclonal antibodies (TNFi), attenuating their clinical efficacy. We tried to determine whether the therapeutic efficacy is different between TNFi with or without the immunoglobulin Fc portion in rheumatoid arthritis (RA) patients depending upon the RF titres.

Methods: RA patients treated with TNFi were enrolled from an observational multi-centre registry in Japan. TNFi treatments were divided into two groups based on the structural characteristics between TNFi with Fc (infliximab, adalimumab, golimumab, etanercept) and TNFi without Fc (certolizumab-pegol). Patients were classified into 4 groups according to RF titre quartiles. The sequential disease activity score in 28 joints using erythrocyte sedimentation rate (DAS28-ESR) were compared by the Mann-Whitney U test between TNFi with and without the immunoglobulin Fc portion in each RF titre group.

Results: A total of 705 RA patients were included and classified into four groups according to quartiles of baseline RF titres (RF ; RF 0-15.0 $\mathrm{IU} / \mathrm{mL}, \mathrm{RF}^{2}$; 15.0-55.0, RF 3 ; 55.0-166, RF ${ }^{4}$; 166-7555). In RF ${ }^{4}$, RA patients treated with TNFi without Fc had a significantly lower DAS28-ESR than those treated with TNFi with Fc (3.2 (2.3-4.2) vs. 2.7 (2.0-3.0)) (median, IQR) after 12 months of follow-up, while there were no significant differences in $\mathrm{RF}^{1}, \mathrm{RF}^{2}$, and $\mathrm{RF}^{3}$.

Conclusion: TNFi without Fc (certolizumab-pegol) may be more efficacious than TNFi with Fc in RA patients with high RF titres.

\section{Background}

Rheumatoid arthritis (RA) is a chronic autoimmune condition with synovitis. The treatment strategy for RA has totally changed since biological disease-modifying antirheumatic drugs (bDMARDs) emerged in clinical practice. Anti-TNF inhibitors (TNFi) were the first bDMARDs introduced in RA clinical practice. To date, five TNFi have been approved for RA treatment, including infliximab (IFX), etanercept (ETN), adalimumab (ADA), golimumab (GLM), and certolizumab-pegol (CZP). However, it is not fully known which TNFi have superior clinical efficacy over other TNFi in patients under specific clinical conditions. The five TNFi have different structural characteristics. IFX, ADA, and GLM are fully functional IgG1 monoclonal antibodies against TNF and contain the immunoglobulin Fc portion, with IFX being a chimeric antibody and ADA and GLM being fully humanized monoclonal antibodies. ETN is a soluble receptor against TNF fused with the Fc portion of human IgG1, and CZP is a monovalent Fab fragment against TNF fused with polyethylene glycol (PEG). Because CZP is a PEGylated Fab fragment, it lacks the Fc portion of immunoglobulin, while all other TNFi (IFX, ADA, GLM, and ETN) have an immunoglobulin Fc portion in their structures [1]. These structural differences in TNFi may lead to differential clinical responses against RA in patients with specific clinical characteristics. However, few studies have analysed the differential clinical responses among different TNFi.

Rheumatoid factor (RF) is an IgM autoantibody against the Fc portion of immunoglobulin G (IgG). High RF titres are the major poor prognostic factors for RA according to the 2019 EULAR recommendations [2]. Because RF can bind to the immunoglobulin Fc portion, it might bind the Fc portion of certain TNFi and influence their clinical efficacy. Therefore, it is possible that high titres of RF might attenuate the clinical effect of those TNFi containing the immunoglobulin Fc portion (TNFi-w/ Fc; IFX, ADA, GLM, and ETN) but not that of TNFi without Fc (TNFi-w/o Fc; CZP). However, no study has compared the clinical efficacy of TNFi with or without the immunoglobulin Fc portion according to RF titres.

In this study, we tried to investigate whether the therapeutic efficacy is different between TNFi with and without the Fc portion in RA patients depending on RF titres, particularly those with high RF titres. To this end, we analysed the data from the ANSWER cohort, a multi-centre registry of RA patients in Japan, which has sequential clinical data before and after bDMARD treatment $[3,4]$.

\section{Materials \& Methods}

\section{Patients}

The Kansai Consortium for Well-being of Rheumatic Disease Patients (ANSWER) cohort is an observational multi-centre registry of patients with RA in the Kansai district of Japan. Data from patients at seven institutes (Kyoto University, Osaka University, Osaka Medical College, Kansai Medical University, Kobe University, Nara Medial University, and Osaka Red Cross Hospital) were included. Patients with RA treated with one of five TNFi (ADA, CZP, ETN, GLM, and IFX; including both intravenous and subcutaneous agents but excluding bio-similar agents, all of which were introduced between 2011 and 2018) and whose data on baseline RF titres and disease activity were available were included in our study. In this study, patients fulfilled the 2010 RA ACR/EULAR criteria. In addition, age, sex, body mass index, disease activity (disease activity score in 28 joints using erythrocyte sedimentation rate (DAS28-ESR), health assessment questionnaire (HAQ)), disease duration, bDMARD naïve rates, concomitant use rates and doses of methotrexate (MTX) and prednisolone (PSL), RF, and anti-cyclic citrullinated peptide antibody (ACPA) were collected. RF (IgM RF) was measured by latex agglutination nephelometric assay. ACPA were measured by anti-CCP2 ELISA kit. Patients were classified into equal quartiles according to baseline $\mathrm{RF}$ titres $\left(\mathrm{RF}^{1}-\mathrm{RF}^{4}\right)$.

Page $3 / 10$ 


\section{Outcome measures}

DAS28-ESR was assessed before and at 3, 6, and 12 months after TNFi initiation, according to baseline RF concentration quartiles. The differences in DAS28-ESR from baseline (dDAS28-ESR) to 3, 6, and 12 months after TNFi initiation were assessed in the same way. The 1-year overall retention rates were also assessed.

\section{Ethics}

The representative facility of this registry is Kyoto University, and this observational study (not clinical trial) was conducted in accordance with the Declaration of Helsinki and approved by each ethics committee of the seven institutes described above (Kyoto University approval number R0357).

\section{Statistical analysis}

An unpaired $t$-test or the Mann-Whitney $U$ test was used when variables were continuous, and the chi-squared test or Fisher's exact test was used when variables were categorical. To deal with missing DAS28-ESR after TNFi initiation, data were analysed using the last observation carried forward method. All analyses were two-tailed, and $P$ values of < .05 were considered statistically significant. All statistical analyses were performed with JMP Pro 14.0 for Mac (SAS Institute Inc., Cary, NC, USA).

\section{Results}

A total of 705 RA patients treated with TNFi were extracted from the ANSWER cohort database. The patients were classified into four groups according to quartiles of baseline RF titres as follows: $\mathrm{RF}^{1}$, patients with RF 0-15.0 IU/mL; RF ${ }^{2}, 15.0-55.0 ; \mathrm{RF}^{3}, 55.0-166$; and $\mathrm{RF}^{4}, 166-7555$. The number of patients in $\mathrm{RF}^{1}$ was 175 ; in $\mathrm{RF}^{2}, 177$; in $\mathrm{RF}^{3}, 176$; and in $\mathrm{RF}^{4}, 177$. The baseline characteristics of the patients are shown in Table 1. In RF ${ }^{4}$, ACPA titres (133 (36-300) vs. 346 (109-500)) (IU/mL) and HAQ (1.0 (0.5-1.6) vs. 1.5 (1.0-2.2)) were higher in patients with TNFi-w/o Fc than in those with TNFi-w/ Fc.

The sequential DAS28-ESR before and at 3, 6, and 12 months after TNFi treatment and dDAS28-ESR at 3, 6, and 12 months after TNFi are shown in Fig. 1. In $\mathrm{RF}^{1}, \mathrm{RF}^{2}$, and $\mathrm{RF}^{3}$, DAS28-ESR tended to be higher in patients with TNFi-w/o Fc than in those with TNFi-w/ Fc at 3, 6, and 12 months after TNFi initiation (TNFi-w/ Fc vs. TNFi-w/o Fc; 3 months RF ${ }^{1} 3.2$ (2.0-4.5) vs. 2.3 (1.8-3.6), RF 2.7 (1.9-3.8) vs. 2.8 (2.2-3.9), $\mathrm{RF}^{3} 3.2(2.3-4.2)$ vs. 4.2 (3.0-4.8); 6 months $\mathrm{RF}^{1} 2.5$ (1.9-3.7) vs. 2.6 (1.9-3.5), RF 2.5 (1.9-3.7) vs. 2.5 (2.0-3.3), RF 2.9 (2.3-4.0) vs. 3.5 (2.6-4.8); 12 months $\mathrm{RF}^{1} 2.4$ (1.9-3.4) vs. 2.6 (1.8-3.6), $\mathrm{RF}^{2} 2.4$ (1.8-3.6) vs. 2.7 (2.1-3.4), RF 2.7 (2.2-3.6) vs. 3.4 (2.6-4.5)). However, in $\mathrm{RF}^{4}$, DAS28-ESR was lower in patients with TNFi-w/o Fc at 3 and 6 months after treatment (TNFi-w/ Fc vs. TNFi-w/o Fc; 3 months 3.8 (2.6-4.8) vs. 3.0 (2.6-4.0); 6 months 3.3 (2.5-4.5) vs. 2.9 (2.2-3.6)), and they showed significantly lower DAS28-ESR after 12 months of treatment than after treatment with TNFi-w/ Fc (3.2 (2.3-4.2) vs. 2.7 (2.0-3.0)). In RF ${ }^{4}$, dDAS28-ESR of TNFi-w/ Fc and TNFi-w/o Fc were as follows: 3 months $0.5(0-1.6)$ vs. $1.8(0.6-3.2), 6$ months $0.7(0-2.0)$ vs. $1.8(0.6-3.5), 12$ months $1.2(0-2.2)$ vs. 2.7 (1.5-3.5). The 1-year retention rates of TNFiw/ Fc and TNFi-w/o Fc were as follows (\%): RF 53.0 vs. 32.4, RF 65.7 vs. $49.4, \mathrm{RF}^{3} 48.8$ vs. $31.0, \mathrm{RF}^{4} 47.6$ vs. 42.1.

In $\mathrm{RF}^{4}$, we compared each TNFi separately, as shown in Supplementary Table S1 and Figure S1. The DAS28-ESR of CZP was lower than that of any other TNFi after 12 months of treatment (ADA 2.7 (2.2-3.9), CZP 2.7 (2.0-3.0), ETN 3.4 (2.6-4.4), GLM 3.2 (2.3-4.3), IFX 3.1 (1.6-4.1)), although the difference was not significant using the Kruskal-Wallis test.

\section{Discussion}

To the best of our knowledge, this is the first study to compare the efficacy of TNFi with and without the immunoglobulin Fc portion in RA patients according to baseline RF titres. The results revealed that TNFi-w/o Fc was more efficacious than TNFi-w/ Fc in RA patients with high RF titres (RF ${ }^{\text {high }}-$ RA patients).

RF is a typical poor prognostic factor of RA. IgM RF amplifies the production of TNF from macrophages induced by the immune complex containing ACPA $[5,6]$. It has been demonstrated that high titres of RF or higher levels of serum TNF concentrations are associated with higher disease activity [7]. In a post hoc analysis of the RISING study, the baseline TNF concentration was increased in ACPA-positive and RF-positive patients, and those patients had attenuated clinical responses to IFX [6]. Thus, high titres of RF are an important factor that could lead to treatment resistance by TNFi. Establishing an appropriate treatment strategy for RF ${ }^{\text {high }}$-RA patients would be of considerable clinical importance.

However, it is not fully understood how RF affects the clinical efficacy of TNFi. Some reports showed that the presence or high titres of RF can be a predictive marker of unresponsiveness to TNFi, but a meta-analysis failed to confirm this finding [8,9]. RA patients with high baseline IgA 
RF titres were associated with poor clinical response to TNFi, including IFX, ADA, and ETN [10]. Furthermore, RF positivity was associated with a poor DAS28-ESR response to TNFi, including IFX, ADA, ETN and GLM $[9,11]$. However, other reports suggested that neither positivity nor titres of RF were associated with treatment resistance to TNFi [12]. These previous studies were all regarding TNFi-w/ Fc (IFX, ADA, GLM, and ETN), and there have been no data on TNFi-w/o Fc (CZP). Our results suggested that TNFi-w/o Fc, namely, CZP, might be efficacious in treating RF ${ }^{\text {high }}$-RA patients. We have some accountable mechanism for this finding as discussed below.

First, RF might bind to the Fc portion of TNFi and attenuate the complement-dependent cytotoxicity (CDC) activity of TNFi-w/ Fc. CDC activity is an important effector function of TNFi, particularly for fully functional monoclonal antibodies (IFX, ADA, and GLM), because it exerts cytotoxic effects against membrane-bound TNF-expressing cells. [4, 13]. Additionally, the Fc portion is known to be required for CDC activity. Because RF binds to the Fc portion of immunoglobulin, RF interferes with the CDC activity of the anti-B cell monoclonal IgG1 antibody rituximab (RTX) in an Fc-dependent manner [14]. High RF titres were associated with reduced CDC activity by RTX in vitro [14]. Considering these reports, RF might bind to the Fc portion of TNFi-w/ Fc and attenuate their therapeutic effect.

Second, RF might affect the neonatal Fc receptor (FcRn)-mediated recycling of monoclonal antibodies, and the serum concentration of TNFi-w/ Fc might be reduced in RF ${ }^{\text {high }}$-RA patients. The in vivo kinetics of IgG are controlled by FcRn, and serum concentrations of monoclonal antibodies with Fc portions are controlled via FcRn-mediated recycling [15]. Circulating IgG in the blood enters monocytes or endothelial cells via fluid phase pinocytosis and is encapsulated into endosomes. Inside endosomes, IgG binds to FcRn and is recycled via exocytosis, while IgG, which cannot be recognized by FcRn, is sorted and degraded by lysosomal functions [16]. Notably, in patients who have the IgG1 allotype G1 $\mathrm{m} 17,1$, IFX competes with their own IgG1 and cannot undergo the recycling process, resulting in a shortened half-life of IFX [17]. RF binds to monoclonal antibodies in the same region of the FcRn binding portion, the $\mathrm{CH} 2-\mathrm{CH} 3$ interface region, and thus RF-bound antibodies cannot undergo the recycling process sufficiently $[18,19]$. Therefore, the half-lives of TNFi-w/ Fc might be shortened in patients with high RF titres, and higher doses of TNFi may be needed to control RA disease activity in RF ${ }^{\text {high }}$-RA patients [6].

CZP is a monovalent Fab' fragment with PEGylation and is the only TNFi that does not possess the immunoglobulin Fc portion [1]. This unique structure allows the therapeutic effects and pharmacokinetics of CZP to be unaffected by Fc-mediated CDC or FcRn-mediated recycling processes [20]. TNFi-w/ Fc may have similar clinical efficacy and pharmacokinetics even in patients with high RF titres. This might have led to the beneficial effect of CZP over other TNFi-w/ Fc in RF ${ }^{\text {high }}$-RA patients.

This study has some limitations, as it is a retrospective cohort study, and possible confounding factors could not be extracted. While baseline characteristics of TNFi-w/ Fc and TNFi-/wo Fc were different about RF titre, ACPA titre, and HAQ in RF ${ }^{4}$, it was not advantage but disadvantage for TNFi-w/o Fc. Therefore, it was still applicable that TNFi-wo Fc was beneficial for RF ${ }^{\text {high }}$ RA patients. We also cannot exclude the possibility that our result is drawn from some unknown confounding factors. In addition, we could not perform adjusted statistical analysis with baseline clinical characteristics due to the small number of patients. Thus, future analysis is required. Mechanistic studies that analyse the binding between RF and the Fc portion of TNFi are also needed.

\section{Conclusions}

In conclusion, our analysis suggested that CZP can be a better treatment option in RF ${ }^{\text {high }}$-RA patients than other TNFi-w/ Fc. Our findings may help promote precision medicine in RA, especially in RF high-RA patients.

\section{List Of Abbreviations}

ACPA: anti-cyclic citrullinated peptide antibody; ADA: adalimumab; bDMARDs: biological disease-modifying antirheumatic drugs; CDC: complement-dependent cytotoxicity; CZP: certolizumab-pegol; DAS28-ESR: disease activity score in 28 joints using erythrocyte sedimentation rate; dDAS28-ESR: the differences in DAS28-ESR; ETN: etanercept; FcRn: neonatal Fc receptor; GLM: golimumab; HAQ: health assessment questionnaire; IFX: infliximab; IgG: immunoglobulin G; MTX: methotrexate; PEG: polyethylene glycol; PSL: prednisolone; RA: Rheumatoid arthritis; RF: rheumatoid factor; RF ${ }^{\text {high }}$-RA patients: RA patients with high RF titres; TNFi: anti-TNF inhibitors; TNFi-w/ Fc: TNFi containing the immunoglobulin Fc portion; TNFi-w/o Fc: TNFi without Fc

\section{Declarations}

\section{Ethics approval and consent to participate}

The representative facility of this registry is Kyoto University, and this observational study was conducted in accordance with the Declaration of Helsinki, with approval by each ethics committee of seven institutes (Kyoto University, Osaka University, Osaka Medical College, Kansai Medical 
University, Kobe University, Nara Medical University, and Osaka Red Cross Hospital). This study was approved by the Institutional Ethical Review Board of Kyoto University Graduate School of Medicine (Kyoto University approval number R0357).

\section{Consent for publication}

Not applicable.

\section{Availability of data and materials}

The datasets used and/or analyzed in the current study are available from the corresponding author on reasonable request.

\section{Competing interests}

M.H., K. Murata, M.T., and H.I belong to Department of Advanced Medicine for Rheumatic Diseases that is supported by Nagahama City, Shiga, Japan, Toyooka City, Hyogo, Japan and five pharmaceutical companies (Mitsubishi Tanabe Pharma Co., Chugai Pharmaceutical Co. Ltd, UCB Japan Co. Ltd, AYUMI Pharmaceutical Co. and Asahi Kasei Pharma Corp.). M.H. has received a research and/or speaker fee from Bristol-Myers, Eisai, Eli Lilly, and Tanabe-Mitsubishi. M.T. has received research grants and/or speaker fees from AbbVie GK, Asahi-Kasei Pharma Corp., Astellas Pharma Inc., Ayumi Pharmaceutical Corp., Bristol-Myers Squibb, Chugai Pharmaceutical Co., Ltd., Eisai Co., Ltd., Eli Lilly Japan K.K., Pfizer Inc., UCB Japan Co., Ltd., Janssen Pharmaceutical K.K., Mitsubishi-Tanabe Pharma Corp., Novartis Pharma K.K., and Taisho Pharma Co., Ltd. H.I. received a research grant and/or speaker fee from Bristol-Myers, Kyocera, Asahi-Kasei and Eisai. KE is affiliated with the Department of Musculoskeletal Regenerative Medicine, Osaka University, Graduate School of Medicine, which is supported by Taisho. KE has received research grants from Abbie, Asahi-Kasei, Astellas, Chugai, Eisai, Ono Pharmaceutical, and UCB Japan. KE has received payments for lectures from Abbvie, Asahi-Kasei, Astellas, Ayumi, Bristol-Myers Squibb, Chugai, Eisai, Eli Lilly, Janssen, Mitsubishi-Tanabe, Ono Pharmaceutical, Sanofi, and UCB Japan. AO received a speaker fee from Chugai, Ono Pharmaceutical, Eli Lilly, Mitsubishi-Tanabe, Asahi-Kasei, and Takeda. RH received a speaker fee from AbbVie. KO received a research grant and/or speaker fee from Abbvie, Astellas, Bristol-Myers Squibb, Eli Lily, MitsubishiTanabe, Pfizer, and Takeda. The remaining authors have no financial conflicts of interest to disclose concerning this manuscript. The pharmaceutical companies had no role in the design of the study, the collection or analysis of the data, the writing of the manuscript, or the decision to submit the manuscript for publication.

\section{Funding}

The study reported in this publication uses the ANSWER cohort supported by grants from ten pharmaceutical companies (AbbVie G.K., AsahiKasei Pharma, AYUMI Pharmaceutical Co., Chugai Pharmaceutical Co., Ltd., Eisai Co., Ltd., Janssen Pharmaceutical K.K., Ono Pharmaceutical Co., Sanofi, Teijin Healthcare Limited, and UCB Japan Co., Ltd.) and an information technology services company (CAC). This study is conducted as an investigator-initiated study. These companies have no roles in the study design, data collection, data analysis, data interpretation or writing of the report.

\section{Author's contributions}

YN was responsible for the conception and design. YN, MH, KMurakami, KMurata, MT, HI, KE, KH, YH, MK, YS, HA, KA, AO, RH, KO, and SM contributed to the data extraction and interpretation. $\mathrm{YN}, \mathrm{WY}$, and $\mathrm{KY}$ contributed to the design and conduction of statistical analysis. $\mathrm{YN}$ and $\mathrm{MH}$ prepared the manuscript. MH supervised the manuscript. The authors read and approved the final manuscript.

\section{Acknowledgements}

The authors thank all medical staff at all institutions participating in the ANSWER cohort study for providing data.

\section{Authors' information}

${ }^{1}$ Department of Rheumatology and Clinical Immunology, Kyoto University Graduate School of Medicine, Japan

${ }^{2}$ Department of Advanced Medicine for Rheumatic Diseases, Kyoto University Graduate School of Medicine, Kyoto, Japan

${ }^{3}$ Department of Health Information Management, Kurashiki Sweet Hospital, Okayama, Japan

${ }^{4}$ Department of Musculoskeletal Regenerative Medicine, Osaka University Graduate School of Medicine, Osaka, Japan

${ }^{5}$ Department of Internal Medicine IV, Division of Rheumatology, Osaka Medical College, Osaka, Japan

${ }^{6}$ Department of Rheumatology, Osaka Red Cross Hospital, Osaka, Japan 
${ }^{7}$ First Department of Internal Medicine, Kansai Medical University, Osaka, Japan

${ }^{8}$ Department of Rheumatology and Clinical Immunology, Kobe University Graduate School of Medicine, Hyogo, Japan

${ }^{9}$ The Center for Rheumatic Diseases, Department of Orthopaedic Surgery, Nara Medical University, Nara, Japan

${ }^{10}$ Department of Medical Informatics, Wakayama Medical University, Wakayama, Japan

\section{References}

1. Bourne T, Fossati G, Nesbitt A. A PEGylated Fab' fragment against tumor necrosis factor for the treatment of Crohn disease: exploring a new mechanism of action. BioDrugs. 2008;22:331-7.

2. Smolen JS, Landewe RBM, Bijlsma JWJ, Burmester GR, Dougados M, Kerschbaumer A, et al. EULAR recommendations for the management of rheumatoid arthritis with synthetic and biological disease-modifying antirheumatic drugs: 2019 update. Ann Rheum Dis. 2020;79:685-99.

3. Ebina K, Hashimoto M, Yamamoto W, Hirano T, Hara R, Katayama M, et al. Drug tolerability and reasons for discontinuation of seven biologics in 4466 treatment courses of rheumatoid arthritis-the ANSWER cohort study. Arthritis Res Ther. 2019;21:91.

4. Hashimoto M, Furu M, Yamamoto W, Fujimura T, Hara R, Katayama M, et al. Factors associated with the achievement of biological diseasemodifying antirheumatic drug-free remission in rheumatoid arthritis: the ANSWER cohort study. Arthritis Res Ther. 2018;20:165.

5. Laurent L, Anquetil F, Clavel C, Ndongo-Thiam N, Offer G, Miossec P, et al. IgM rheumatoid factor amplifies the inflammatory response of macrophages induced by the rheumatoid arthritis-specific immune complexes containing anticitrullinated protein antibodies. Ann Rheum Dis. 2015;74:1425-31.

6. Takeuchi T, Miyasaka N, Inui T, Yano T, Yoshinari T, Abe T, et al. High titers of both rheumatoid factor and anti-CCP antibodies at baseline in patients with rheumatoid arthritis are associated with increased circulating baseline TNF level, low drug levels, and reduced clinical responses: a post hoc analysis of the RISING study. Arthritis Res Ther. 2017;19:194.

7. Takeuchi T, Miyasaka N, Tatsuki Y, Yano T, Yoshinari T, Abe T, et al. Baseline tumour necrosis factor alpha levels predict the necessity for dose escalation of infliximab therapy in patients with rheumatoid arthritis. Ann Rheum Dis. 2011;70:1208-15.

8. Salgado E, Maneiro JR, Carmona L, Gomez-Reino J. Rheumatoid factor and response to TNF antagonists in rheumatoid arthritis: systematic review and meta-analysis of observational studies. Joint Bone Spine. 2014;81:41-50.

9. Potter C, Hyrich KL, Tracey A, Lunt M, Plant D, Symmons DP, et al. Association of rheumatoid factor and anti-cyclic citrullinated peptide positivity, but not carriage of shared epitope or PTPN22 susceptibility variants, with anti-tumour necrosis factor response in rheumatoid arthritis. Ann Rheum Dis. 2009;68:69-74.

10. Bobbio-Pallavicini F, Caporali R, Alpini C, Avalle S, Epis OM, Klersy C, et al. High IgA rheumatoid factor levels are associated with poor clinical response to tumour necrosis factor alpha inhibitors in rheumatoid arthritis. Ann Rheum Dis. 2007;66:302-7.

11. Santos-Moreno P, Sanchez G, Castro C. Rheumatoid factor as predictor of response to treatment with anti-TNF alpha drugs in patients with rheumatoid arthritis: results of a cohort study. Medicine. 2019;98:e14181.

12. Lequerre T, Jouen F, Brazier M, Clayssens S, Klemmer N, Menard JF, et al. Autoantibodies, metalloproteinases and bone markers in rheumatoid arthritis patients are unable to predict their responses to infliximab. Rheumatology. 2007;46:446-53.

13. Hirosaki H, Maeda Y, Shimojima M, Maeda K, Iwata H, Takeyoshi M. Effects of soluble tumor necrosis factor (TNF) on antibody-dependent cellular cytotoxicity of therapeutic anti-TNF-alpha antibody. Immunol Invest. 2019;48:441-50.

14. Jones JD, Shyu I, Newkirk MM, Rigby WF. A rheumatoid factor paradox: inhibition of rituximab effector function. Arthritis Res Ther. 2013;15:R20.

15. Liu L. Pharmacokinetics of monoclonal antibodies and Fc-fusion proteins. Protein Cell. 2018;9:15-32.

16. Ward ES, Ober RJ. Targeting FcRn to generate antibody-based therapeutics. Trends Pharmacol Sci. 2018;39:892-904.

17. Ternant D, Arnoult C, Pugniere M, Dhommee C, Drocourt D, Perouzel E, et al. IgG1 allotypes influence the pharmacokinetics of therapeutic monoclonal antibodies through FcRn binding. J Immunol. 2016;196:607-13.

18. Artandi SE, Calame KL, Morrison SL, Bonagura VR. Monoclonal IgM rheumatoid factors bind IgG at a discontinuous epitope comprised of amino acid loops from heavy-chain constant-region domains 2 and 3. Proc Natl Acad Sci U S A. 1992;89:94-8.

19. Roopenian DC, Akilesh S. FcRn: the neonatal Fc receptor comes of age. Nat Rev Immunol. 2007;7:715-25.

20. Porter C, Armstrong-Fisher S, Kopotsha T, Smith B, Baker T, Kevorkian L, et al. Certolizumab pegol does not bind the neonatal Fc receptor (FcRn): consequences for FcRn-mediated in vitro transcytosis and ex vivo human placental transfer. J Reprod Immunol. 2016;116:7-12. 


\section{Tables}

Table 1. Baseline patient characteristics in each group $\left(\mathrm{RF}^{1}-\mathrm{RF}^{4}\right)$.

Baseline characteristics were compared between RA patients treated with TNF inhibitors with the Fc portion (w/Fc) and those without (w/o Fc).

Continuous data were analysed using an unpaired $t$-test or the Mann-Whitney $U$ test, and categorical data were analysed using the chi-squared test or Fisher's exact test. 


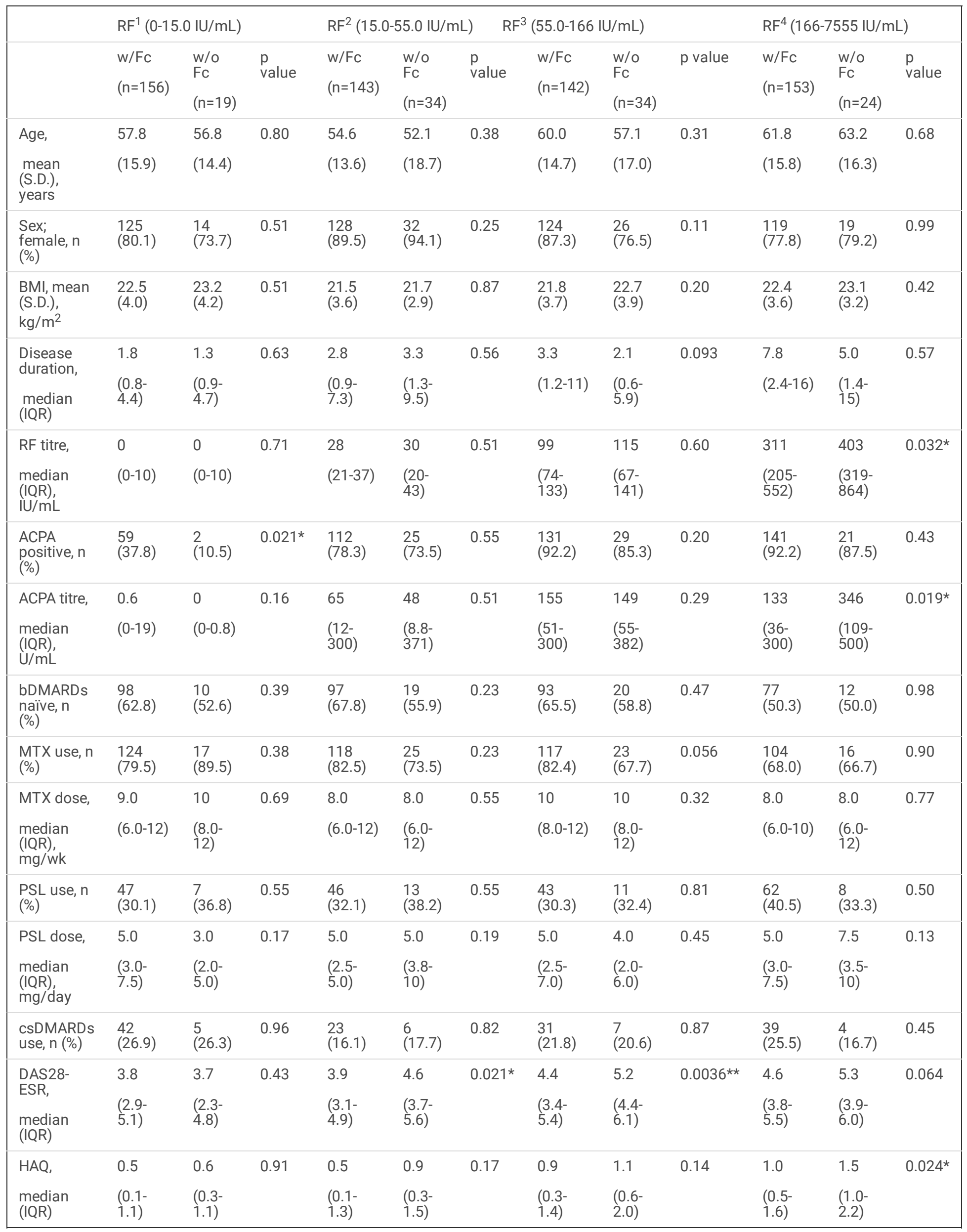


ACPA: anti-cyclic citrullinated peptide antibody; bDMARDs: biological disease-modifying antirheumatic drugs; BMl: body mass index; csDMARDs: conventional synthetic disease-modifying antirheumatic drugs (bucillamine, iguratimod, leflunomide, salazosulfapyridine, tacrolimus); DAS28-ESR: disease activity score in 28 joints using erythrocyte sedimentation rate; HAQ: health assessment questionnaire; IQR: inter quartile range; MTX: methotrexate; PSL: prednisolone; RF: rheumatoid factor; SD: standard deviation.

$\star p<0.05 * * p<0.01$

\section{Figures}

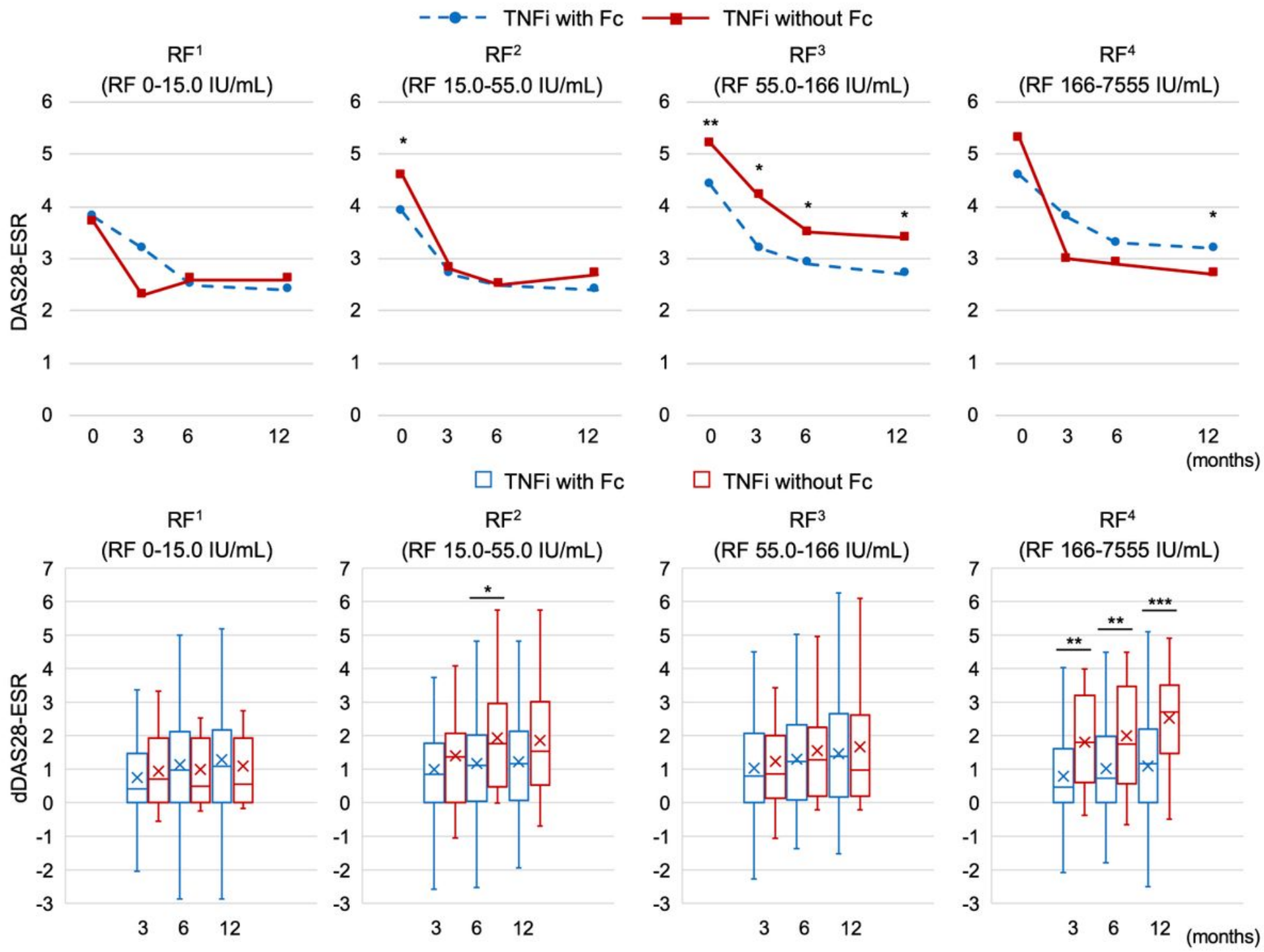

Figure 1

The graphs of disease activity score in RF1 - RF4. (Upper tier) Disease activity score in 28 joints using erythrocyte sedimentation rate (DAS28ESR) before and at 3, 6, and 12 months after TNF inhibitor (TNFi) initiation. Median. (Lower tier) The differences in DAS28-ESR between baseline and 3, 6, and 12 months after TNFi initiation. Data were shown by median with interquartile range. Each variable was compared using the Mann-Whitney U test. RF: rheumatoid factor. ${ }^{*} p<0.05$, ${ }^{\star \star} p<0.01,{ }^{\star * \star} p<0.001$

\section{Supplementary Files}

This is a list of supplementary files associated with this preprint. Click to download.

- 20200818FigureS1.png

- 20200818TableS1.docx 